\title{
Rapid method for the quantification of reduced and oxidized glutathione in human plasma and saliva
}

\author{
Kamonwad Ngamchuea, Christopher Batchelor-McAuley, Richard G. Compton*
}

*corresponding author: Richard G. Compton, Department of Chemistry, Physical \& Theoretical Chemistry Laboratory, University of Oxford, South Parks Road, Oxford, OX1 3QZ, United Kingdom

Email: richard.compton@ chem.ox.ac.uk. Tel: +44(0)1865275957 Fax: +44(0)1865275410

\begin{abstract}
A new method is developed to determine the concentrations of reduced (GSH) and oxidized glutathione (GSSG), and hence the GSH:GSSG ratios in human plasma and saliva samples. The assay is based on the masking of GSH in a GSH and GSSG mixture via a 1,4-addition reaction with $p$-benzoquinone (BQ), followed by enzymatic kinetic measurement. The enzyme, glutathione reductase, is highly specific to glutathione. Excess BQ can thus be easily removed by the addition of non-GSH thiols. The assay takes less than $2 \mathrm{~min}$, is suitable for short timescale study and minimizes the in-vitro underestimation of the GSH:GSSG ratio arising from the degradation of GSH and formation of GSSG. We further show in this paper that the stability of the total glutathione content (GSH + GSSG) and GSH in saliva is significantly greater than in plasma, encouraging the development of non-invasive saliva sensing.
\end{abstract}




\section{Introduction}

An increase in the level of prooxidants, notably reactive oxygen species, relative to that of antioxidants in cells and tissues results in 'oxidative stress' which potentially leads to oxidative damage to important biological components such as DNA, proteins and lipids. ${ }^{1}$ The level of oxidative stress can be assessed using marker compounds. Among those, glutathione is one of the most widely-used indicators. The reduced form of glutathione, GSH, is an important water-soluble antioxidant and the most abundant thiol compound in the human body. The change in the ratio of reduced (GSH) to oxidized glutathione (GSSG) has been reported to be diagnostic of various diseases such as cancers, ${ }^{2}$ neurodegenerative diseases, ${ }^{3}$ and cardiovascular diseases. ${ }^{4}$ Considering the valuable information that can be extracted, the primary aim of this paper is to evaluate the levels of GSH, GSSG and hence the ratio between the two species in biological samples including human plasma and saliva. Plasma is commonly used for health diagnosis, while saliva is a possible superior alternative offering the advantage of non-invasive sample collection. Saliva is particularly suitable for point-ofcare analysis as well as for those with hemophobia and trypanophobia.

Plasma glutathione is known to decrease at a significant rate at room temperature in-vitro with a half-life of approximately 20 min. ${ }^{6}$ However, there is no report on the stability of salivary glutathione at room temperature. For diagnostic analysis, it is important that the concentrations of glutathione are accurately determined and reflect the 'true' concentrations in the human body. It is therefore favourable to use the biological medium which exhibits the slowest rate of glutathione degradation. To evaluate the reliability of glutathione concentrations measured as well as to explore the possibility of using saliva as a diagnostic medium, kinetic and recovery studies are performed in this paper. The stabilities of total glutathione and GSH in plasma and saliva samples are investigated alongside the formation of GSSG, for which a new methodology is developed. 
At present, the contents of GSH and GSSG in biological samples can be determined by various means notably chromatographic methods (HPLC, GC), ${ }^{7,8}$ capillary electrophoresis, ${ }^{9,10}$ electrochemical techniques, ${ }^{11-13}$ nanoparticle-based colorimetric tests,${ }^{14}$ chemiluminescence, ${ }^{15}$ spectrofluorimetric methods and spectrophotometric methods. ${ }^{16,17}$ Among those, one of the most commonly used methods is the spectrophotometric enzymatic assay first developed by Tietze. ${ }^{18}$ This colorimetric test is based on the thiol-disulfide exchange reaction between GSH and the sulfhydryl reagent 5,5'-dithiobis(2-nitrobenzoic acid) (DTNB). The reaction produces a chromophore that strongly absorbs at the wavelength of $412 \mathrm{~nm}$ (5-thio-2nitrobenzoate, $\mathrm{TNB}^{2-}$ ) and a mixed disulfide $\mathrm{GS}_{-} \mathrm{TNB}^{-} . \mathrm{GS}_{-} \mathrm{TNB}^{-}$is then reduced by the enzyme glutathione reductase (GR) to form another $\mathrm{TNB}^{2-}$ and regenerate $\mathrm{GSH}$. The recycle rate of GSH, which is proportional to the GSH level, is then measured by monitoring the rate of $\mathrm{TNB}^{2-}$ formation at $412 \mathrm{~nm}$. The method finds widespread use in part due to its low cost, convenience, high sensitivity, and low limit of detection of ca. $10 \mathrm{nM}$. The Tietze assay however measures the concentration of total glutathione, both reduced and oxidized forms, due to the catalytic action of the enzyme.

To determine the levels of GSH and GSSG, and hence the ratio between the two species, an extra step of masking of GSH is required prior to the enzymatic reactions. Currently, there are two widely-used thiol-masking reagents for uses in the enzymatic assay, $\mathrm{N}$ ethylmaleimide $(\mathrm{NEM}){ }^{18}$ and 2-vinylpyridine $(2 \mathrm{VP}),{ }^{19}$ each of which has its own advantages and disadvantages. The former reacts with GSH almost instantaneously but is highly toxic and known to inhibit the activity of glutathione reductase used in the measurement assay. Consequently, any excess NEM has to be removed by column chromatography ${ }^{20}$ or by multiple extractions with organic solvents ${ }^{18,21}$ before performing the enzymatic cycling. This has led to the development of another thiol-masking agent, 2VP, which does not inhibit the enzyme, but nonetheless, has its own disadvantage of a very slow reaction with GSH. In 
particular, it takes ca. 20 - 60 min for the reaction between GSH and 2VP to go to completion and hence fully mask all thiols from the sample.

In this paper, we explore a new GSH-masking reagent based on benzoquinone (BQ). BQ is much less toxic than NEM, and efficiently and almost instantaneously binds to the target thiol. More importantly, any excess BQ can be easily and quickly removed $(<20 \mathrm{~s})$ by the method developed herein. It is thus a significant improvement as compared to the original NEM or 2VP assays, allowing rapid detection of both GSH and GSSG in biological samples.

\section{Experimental Section}

This section provides the experimental details of the reagents used, the sources and storage of the biological samples as well as the procedures for the measurements of GSH and GSSG, and the stability studies. More specific details such as the concentrations of stock solutions and the amount of solutions used are provided in the SI.

\subsection{Chemical reagents and instrumentation}

All reagents were purchased from Sigma-Aldrich and were used as received without further purification: L-glutathione reduced (GSH, $\geq 98.0 \%$ ), L-Glutathione oxidized (GSSG, $\geq 98.0 \%$ ), 5,5'-dithiobis(2-nitrobenzoic acid) (DTNB, $\geq 98 \%$ ), ethylenediaminetetraacetic acid disodium salt dehydrate (EDTA, 99.0-101.0\%), $\beta$-nicotinamide adenine dinucleotide 2'phosphate reduced tetrasodium salt hydrate (NADPH, $\geq 97 \%$ ), glutathione reductase from baker's yeast (S. cerevisiae) (GR, 100-300 units/mg protein (biuret)), 1,4-benzoquinone (BQ), 1,4-naphthoquinone (97\%), 2-chloro-1,4-benzoquinone (95\%), 2,6-dichloroquinone-4chloroimide, sodium phosphate dibasic $\left(\mathrm{Na}_{2} \mathrm{HPO}_{4}, \geq 99.0 \%\right)$ and sodium phosphate monobasic dihydrate $\left(\mathrm{NaH}_{2} \mathrm{PO}_{4} \cdot 2 \mathrm{H}_{2} \mathrm{O}, \geq 99.0 \%\right)$. All solutions were prepared using deionized water (Millipore) with a resistivity of $18.2 \mathrm{M} \Omega \mathrm{cm}$, and all the reaction mixtures thermostated at $25^{\circ} \mathrm{C}$. The measurements were performed with a Shimadzu UV-1800 UV-Vis 
spectrophotometer in disposable cuvettes (Eppendorf UVette, Sigma-Aldrich) using a $10 \mathrm{~mm}$ optical path length.

\subsection{Biological samples}

Plasma samples were supplied by ZenBio (NC, USA). The plasma was collected in acid citrate dextrose from consenting adult (male) donors with the average age of 36.5 (range: 2057). The plasma was shipped frozen on dry ice and stored at $-80{ }^{\circ} \mathrm{C}$ prior to use. ${ }^{22}$ Saliva samples were kindly donated by laboratory colleagues without any known pathological conditions. Saliva was taken at least 1 hour after fasting using Salivette ${ }^{\circledR}$ and immediately stored at $-20{ }^{\circ} \mathrm{C} .{ }^{23}$ The samples were then used within 2 hours after collection.

\subsection{Measurement procedures}

The concentrations of total glutathione (in GSH equivalents; [GSH] + 2[GSSG]) are measured using the Tietze enzymatic kinetic assay. ${ }^{18}$ The Tietze assay is based on the reaction between GSH and 5,5'-Dithiobis(2-nitrobenzoic acid) (DTNB). The reaction produces 5-thio-2-nitrobenzoate $\left(\mathrm{TNB}^{2-} ; \quad \varepsilon \lambda=412 \mathrm{~nm}=14,150 \mathrm{M}^{-1} \mathrm{~cm}^{-1}\right)^{24}$ and a mixed disulfide $\left(\mathrm{GS}-\mathrm{TNB}^{-}\right)$. GS-TNB ${ }^{-}$is then reduced by glutathione reductase $(\mathrm{GR})$ in the presence of NADPH to form another $\mathrm{TNB}^{2-}$ and GSH. Any GSSG present in the sample is converted to GSH by GR at the beginning of the assay.

Subsequently, an aliquot of the sample is added to the solutions of DTNB and GR. After $30 \mathrm{~s}$, NADPH is added to the solution mixtures. The absorbance at $412 \mathrm{~nm}\left(\mathrm{~A}_{412}\right)$ is then recorded for $120 \mathrm{~s}$ and the slope of $\mathrm{A}_{412}$ against time $\left(d \mathrm{~A}_{412} / d t\right)$, which is linearly proportional to the concentration of total glutathione in the sample, is determined.

At the same time, the concentrations of oxidized glutathione (GSSG) are determined using the modified enzymatic assay developed in this work, referred to as the $G S S G\left(B Q / T N B^{2-}\right)$ 
assay. First, benzoquinone (BQ) is used to mask GSH in the samples. After the solution is well-mixed, $\mathrm{TNB}^{2-}$ is added to the reaction mixture to remove excess $\mathrm{BQ}$. An aliquot of the mixture is then transferred to a UV-Vis cuvette, followed by the addition of DTNB, GR and $\mathrm{NADPH}$. The spectrophotometric monitoring at $412 \mathrm{~nm}\left(\mathrm{~A}_{412}\right)$ is then performed as described above. The concentrations, temperature and $\mathrm{pH}$ are optimized below and the final protocol is presented in Scheme 1.

The levels of reduced glutathione (GSH) can be calculated from the difference in concentrations of the total and oxidized glutathione.

\subsection{Recovery study in plasma and saliva samples}

Plasma and saliva samples are spiked with $15 \mu \mathrm{M}, 50 \mu \mathrm{M}$ (plasma only) and $100 \mu \mathrm{M}$ GSH in final concentrations. The spiked samples are incubated at $25^{\circ} \mathrm{C}$ and the concentrations of total, reduced and oxidized glutathione determined every $10 \mathrm{~min}$ for the total of $90 \mathrm{~min}$ using the Tietze assay together with the GSSG assay.

\section{$3 \quad$ Results and Discussions}

The GSSG assay developed herein is based on the selected 1,4-addition ("Michael addition") of the GSH thiol in a mixture of GSH and GSSG to a quinone. After reaction, only the oxidized form of glutathione, GSSG, is left in the sample. The quinone-treated sample is then subjected to the Tietze enzymatic kinetic assay, which determines the concentration of the remaining GSSG. The assay is first optimized to explore the most efficient excess quinone scavenger and the thiol-masking quinone, as well as the most suitable reaction conditions: $\mathrm{pH}$, temperature, reaction time and concentrations. The optimized assay is then validated using variable concentrations of GSH, GSSG and mixtures of GSH and GSSG standards. Subsequently, the assay is applied to real biological samples including human plasma and saliva samples. Further applications of the assay to investigate the stability of the total 
glutathione and of GSH, as well as the formation of GSSG in plasma and saliva samples are then performed.

\subsection{Assay optimization}

GSH standard solutions were subjected to the Tietze assay to determine the rate of $\mathrm{TNB}^{2-}$ formation $\left(s_{0}\right)$ for each concentration. Aliquots of the GSH solutions were then reacted with quinone, and the excess quinone removed by the addition of a non-GSH thiol, as detailed later. Afterwards, the quinone-treated solutions were subjected to the enzymatic measurement. The resulting rate of $\mathrm{TNB}^{2-}$ formation $\left(s_{1}\right)$ reveals the amount of GSH left unreacted. The proportion of GSH remained in the solution is calculated by $s_{0} / s_{1} \times 100 \%$. The amount of GSH removed by quinone is therefore $\left(1-s_{0} / s_{1}\right) \times 100 \%$.

Excess thiol-masking quinone in the solution has to be removed prior to the enzymatic measurements to prevent undesirable reactions between quinone and the $\mathrm{TNB}^{2-}$ formed during enzymatic cycling. Due to the selectivity of the enzyme, this can be done by the addition of thiols other than GSH. The quinone-scavenging non-GSH thiol does not contribute to the rate of $d \mathrm{~A}_{412} / d t^{25,26}$

To identify the most suitable quinone-quenching reagent, four thiols which react rapidly with quinone including cysteine, 2-mercaptoethanesulfonate, 2-mercaptoimidazole and $\mathrm{TNB}^{2-}$ were investigated (see Chart S1 in the SI for structures of the compounds). The first three thiols were supplied by commercial providers, while $\mathrm{TNB}^{2-}$ was made by the addition of $\mathrm{NaBH}_{4}$ to the solution of DTNB (SI). The solution of $40 \mu \mathrm{M}$ GSH was treated with $300 \mu \mathrm{M}$ benzoquinone, followed by $300 \mu \mathrm{M}$ of the different thiols. The concentration of the remaining GSH was determined to find out the most efficient quinone-quenching reagent. In addition to GSH standards, the reactions between $20 \mu \mathrm{M}$ GSSG and $300 \mu \mathrm{M}$ benzoquinone, 
followed by $300 \mu \mathrm{M}$ of the thiols were performed to determine whether the thiols added interferes with the measurement assay.

It was found that among the four thiols studied, $\mathrm{TNB}^{2-}$ allowed optimal scavenging of quinone that when tested with GSH solutions, $1.4 \pm 0.1 \%$ of GSH remains and when tested with GSSG solutions, $>99 \%$ of GSSG is recovered. $\mathrm{TNB}^{2-}$ is thus used as a quinonescavenger throughout the remainder of this work. The results for the other thiols studied are provided in the SI. Note that the concentration of GSSG is determined from the 'rate' of $\mathrm{TNB}^{2-}$ formation $\left(d \mathrm{~A}_{412} / d t\right.$ ), and any background $\mathrm{TNB}^{2-}$ in the solution (up to $370 \mu \mathrm{M}$ studied in this work) shows no evidence of disturbance to $d \mathrm{~A}_{412} / d t$.

Next, it is important to identify a quinone compound which very efficiently removes free GSH from the system. Four quinone species with different substituents including 1,4benzoquinone, 1,4-naphthoquinone, 2-chloro-1,4-benzoquinone and 2,6-dichloroquinone-4chloroimide were investigated (see Chart S2 in the SI for structures of the compounds). The solutions of $40 \mu \mathrm{M}$ GSH in the presence of $300 \mu \mathrm{M}$ of different quinones (followed by 300 $\mu \mathrm{M} \mathrm{TNB}^{2-}$ ) were studied. The concentration of the remaining GSH was determined to find out the most efficient thiol-masking quinone. The results show that $1.4 \pm 0.1 \%, 22.4 \pm 0.6 \%$, $2.8 \pm 0.1 \%$ and $4.6 \pm 0.3 \%$ of free GSH remained in the solution when 1,4-benzoquinone, 1,4-naphthoquinone, 2-chloro-1,4-benzoquinone and 2,6-dichloroquinone-4-chloroimide were used respectively. It has been further shown that if excess benzoquinone is removed by sufficient amount of $\mathrm{TNB}^{2-}$, GSSG concentration is not affected by the use of the reagent in the assay (SI). Consequently, 1,4-benzoquinone (BQ) was chosen as the thiol-masking agent for the GSSG assay.

The reaction conditions including reaction time (between GSH and $\mathrm{BQ}$ ), $\mathrm{pH}$, temperature and concentrations are optimized next. 
Reaction time. The reaction between BQ and GSH goes to completion in less than $20 \mathrm{~s}$. Increasing the reaction time does not result in further masking of GSH i.e. no change in the $1.5 \pm 0.2 \%$ of GSH remained in the solution. Full results are provided in the SI.

Temperature. The reaction was studied at $0{ }^{\circ} \mathrm{C}, 25^{\circ} \mathrm{C}$ and $40^{\circ} \mathrm{C}$. The amounts of $\mathrm{GSH}$ remained in the three temperatures studied were $1.6 \pm 0.1 \%, 1.4 \pm 0.1 \%$ and $1.7 \pm 0.1 \%$ at $0{ }^{\circ} \mathrm{C}, 25{ }^{\circ} \mathrm{C}$ and $40{ }^{\circ} \mathrm{C}$ respectively, and hence not distinguishably different within experimental errors. For convenience, the assay is therefore suggested to be performed at room temperature $\left(25^{\circ} \mathrm{C}\right)$.

$p H$. The reaction between BQ and GSH was studied at $\mathrm{pH} 1.0,7.5$ and 12.0. At $\mathrm{pH} 7.5$ and 12.0, the masking of GSH was similarly efficient with $1.4 \pm 0.1 \%$ and $1.7 \pm 0.1 \%$ of GSH remained respectively. In contrast, > 44\% of GSH was left unmasked in the solution at $\mathrm{pH}$ 1.0. The inefficient masking in acidic condition is thought to be due to the unfavourable 1,4addition reaction between the protonated TNB and BQ. The higher amount of unmasked GSH equivalent at $\mathrm{pH} 12.0$ as compared to at $\mathrm{pH} 7.5$ is likely due to either the formation of a small amount of GSSG at very high $\mathrm{pH}^{27}$ or the reaction of $\mathrm{OH}^{-}$with $\mathrm{BQ}{ }^{28}$ The authors therefore suggest the reaction medium to be at $\mathrm{pH} \sim 7.5$ for the most efficient masking of GSH and so that additional $\mathrm{pH}$ adjustment when performing the enzymatic cycling is not required.

Concentrations. For $40 \mu \mathrm{M}$ of GSH, $50 \mu \mathrm{M}$ of BQ has been observed to be able to minimize the amount of remaining unmasked GSH to $1.5 \pm 0.3 \%$, the lowest we can possibly obtain using the developed assay. Increasing the concentration of BQ further does not change the amount of GSH left in the solution. At the same time, the concentration of $\mathrm{TNB}^{2-}$ has to be in excess of any remaining unreacted $\mathrm{BQ}$ to ensure none of $\mathrm{BQ}$ is left to interfere with the formation of $\mathrm{TNB}^{2-}$ from enzymatic cycling. 


\subsection{Assay validation and standard curves}

In this section, the optimized GSSG $\left(\mathrm{BQ} / \mathrm{TNB}^{2-}\right)$ assay is validated. Standard solutions of variable concentrations: GSH, GSSG and GSH/GSSG mixtures were studied. First, GSH solutions were tested to evaluate whether the $\mathrm{BQ} / \mathrm{TNB}^{2-}$ assay efficiently removes GSH from the solutions. Second, GSSG solutions were studied to ensure that the $\mathrm{BQ} / \mathrm{TNB}^{2-}$ assay selectively masks GSH, and does not affect the concentration of GSSG. Third, the GSH/GSSG solution mixtures were studied to demonstrate that the $\mathrm{BQ} / \mathrm{TNB}^{2-}$ assay, together with the Tietze assay, can be used to quantify the amount of both GSH and GSSG, as well as the ratio between the two species accurately. Consequently, the standard solutions were subjected to the total glutathione (Tietze) and the GSSG assays. The rates of change in the absorbance at $412 \mathrm{~nm}\left(d \mathrm{~A}_{412} / d t\right)$ were measured in the absence of $\mathrm{BQ} / \mathrm{TNB}^{2-}$ (i.e. total glutathione assay) and were compared with those in the presence of the $\mathrm{BQ} / \mathrm{TNB}^{2-}$ (i.e. GSSG assay).

For all the solutions of GSH studied, the results of $d \mathrm{~A}_{412} / d t$, presented as a function of GSH concentration in Figure 1a, show the efficient depletion of free GSH in the BQ/TNB ${ }^{2-}$ assay with less than $1.5 \%$ of GSH remaining in the solution after the reaction with BQ for all the concentrations studied. At the same time, the concentrations of GSSG in standard GSSG solutions were not altered by the presence of $\mathrm{BQ}$ and excess $\mathrm{TNB}^{2-}$ within the experimental errors of $1.8 \%$; refer to Figure 1b. The sensitivity of GSSG measurement obtained in the presence of $50 \mu \mathrm{M}$ BQ and $50 \mu \mathrm{M} \mathrm{TNB}^{2-}$, at the range $0-20 \mu \mathrm{M}$, is $0.307 \pm 0.002 \mathrm{~ms}^{-1}$ $\mu \mathrm{M}^{-1}$. The limit of detection $\left[\mathrm{c}_{\mathrm{L}(\mathrm{k}=3)}\right]$ is determined as defined by IUPAC using the propagation errors approach ${ }^{29}$ to be $0.02 \mu \mathrm{M}$. 
The assay was further applied to the mixtures of GSH and GSSG. The reactions as well as the measurements were performed in buffered solution containing $10 \mathrm{mM}$ EDTA. At this high concentration of EDTA, it has been reported that oxidation of GSH to GSSG in nonbiological media does not occur within 3 hours with the experimental errors of less than $\pm 2 \% .{ }^{30}$ The reference GSH:GSSG ratio in the solution can therefore be taken from the concentrations of GSH and GSSG used to prepare the solution. The ratios of GSH:GSSG measured using the assay are $98.7 \pm 1.3 \%$ of the actual ratios of GSH:GSSG in the solutions, as displayed as a scattered plot in Figure 1c, evidencing the high accuracy of the developed assay.

\subsection{Assay limitation}

As discussed above, there is an inherent error of ca. $1.5 \%$ of the assay, the size of which is comparable to that reported for the $2 \mathrm{VP}$ assay. ${ }^{19}$ The origin of the small amount of remaining GSH equivalent is unclear. One possibility is the conversion of a small amount of GSH to GSSG. Although literature reports suggest that no GSSG is formed from the reaction between BQ and GSH, ${ }^{31,32}$ there may be a difference in the sensitivity of the technique used. Another possible route is the substitution reaction of $\mathrm{TNB}^{2-}$ to the GS-BQ compound, resulting in the reformation of free GSH. However, this process is also unlikely due to $\mathrm{TNB}^{2-}\left(\mathrm{pK}_{\mathrm{a}}\right.$, thiol $=$ $4.5)^{33}$ being a much better leaving group than $\mathrm{GS}^{-}\left(\mathrm{pK}_{\mathrm{a}}\right.$, thiol $\left.=9.1\right) \cdot{ }^{34}$ No GSSG impurity is detected in the standard solutions as determined by titration with DTNB without the enzymatic cycling.

Due to the $1.5 \%$ error of the assay, the measurements of GSH:GSSG ratio is not recommended for systems whose ratios are expected to exceed $\sim 67$. Nonetheless, the concentrations of GSH, GSSG, as well as the ratio between the two species (i.e. oxidative status) in typical biological samples such as plasma and saliva are within the ranges that can 
be detected accurately (within $1.5 \%$ of the measurement error) using the developed assay, as will be evidenced in the following section.
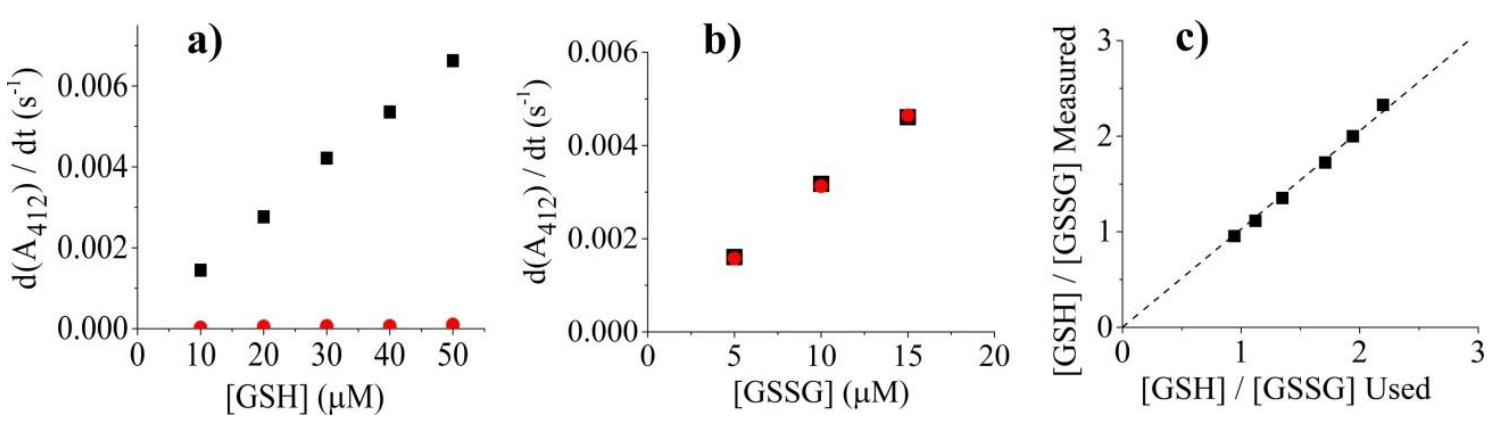

Figure 1: Calibration curves showing the rates of $\mathrm{TNB}^{2-}$ formation $\left(d \mathrm{~A}_{412} / d t / \mathrm{s}^{-1}\right)$ determined by (Black ( $\left.\left.\mathbf{\square}\right)\right)$ Tietze assay using the conditions recommended by Rahman et al. ${ }^{35}$; $(\boldsymbol{R e d}(\bullet))$ the GSSG $\left(\mathrm{BQ} / \mathrm{TNB}^{2-}\right)$ assay using $50 \mu \mathrm{M} \mathrm{BQ}, 50 \mu \mathrm{M} \mathrm{TNB}^{2-}, 25^{\circ} \mathrm{C}, \mathrm{pH} 7.5$ for variable concentrations of the solutions of a) GSH and b) GSSG. c) The GSH:GSSG ratios determined $v s$. the GSH:GSSG ratios used to prepare the solutions.

\subsection{Applications of the GSSG $\left(\mathrm{BQ} / \mathrm{TNB}^{2-}\right)$ assay}

\section{Quantification of GSH and GSSG in human plasma and saliva samples}

The optimized $\mathrm{BQ} / \mathrm{TNB}^{2-}$ assay has been validated against standard GSH and GSSG solutions. In this section, the assay will be demonstrated for its typical uses in the quantification of GSH and GSSG in biological samples. Two biological media are employed. One is the most commonly used for health diagnosis, plasma. The other is the clinically noninvasive alternative saliva.

In this work, the biological samples are assayed without deproteinization. However, if the samples have been deproteinized by the addition of acids, ${ }^{35}$ the $\mathrm{pH}$ has to be adjusted to ca. 7.5 prior to the measurement, simply by the addition of $\mathrm{NaOH}$ as the reaction between $\mathrm{BQ}$ and GSH is not efficient in acidic solution, as mentioned above. In addition, BQ can react with other thiols in the samples such as cysteine, homocysteine, as well as other nucleophiles and reducing agents. Therefore, a sufficient amount of BQ has to be added to ensure that the maximal possible amount of GSH in the sample has been masked by the BQ. For this reason, 
the amount of BQ used in biological samples for the measurement of GSSG is $185 \mu \mathrm{M}$ (followed by $370 \mu \mathrm{M} \mathrm{TNB}^{2-}$ ), higher than the $50 \mu \mathrm{M}$ (followed by $50 \mu \mathrm{M} \mathrm{TNB}^{2-}$ ) used for the standard solutions. The BQ concentration of $185 \mu \mathrm{M}$ employed has been validated by the addition of increasing concentrations of GSH solutions to aliquots of the samples. The concentration of BQ may be adjusted to suit various samples as long as the concentration of $\mathrm{TNB}^{2-}$ is adjusted accordingly. The results are shown in Figures $2 \mathrm{a}$ and $2 \mathrm{~b}$ for plasma and saliva respectively. The errors of the measurement (\%GSH remained) are determined from the slopes of the amount of GSH remaining against the amount of GSH added to be $0.7 \pm 0.1$ $\%$ and $0.1 \pm 0.03 \%$ for plasma and saliva respectively. The amount of GSH equivalent detected when no GSH is added reflects the concentration of GSSG present in the sample.

The assay has then been employed to quantify the amount of GSH and GSSG in plasma and saliva samples. The levels of GSH and GSSG found in plasma were $4.20 \pm 0.12 \mu \mathrm{M}$ and $1.99 \pm 0.08 \mu \mathrm{M}$ respectively, similar to the values reported in literature measured using HPLC. ${ }^{36}$ In saliva, $3.14 \pm 0.50 \mu \mathrm{M}$ and $0.60 \pm 0.15 \mu \mathrm{M}$ were detected for GSH and GSSG respectively, consistent with the literature values determined by HPLC $^{37}$ and by chromatography / mass spectroscopy ${ }^{38}$. The assay has thus been proved to be able to accurately determine the concentrations of GSH and GSSG in plasma and saliva samples. More importantly, the developed technique is significantly quicker and less inexpensive than measurements by HPLC or mass spectroscopy. It also does not require specific training for use of the instrument. 


\section{a)}
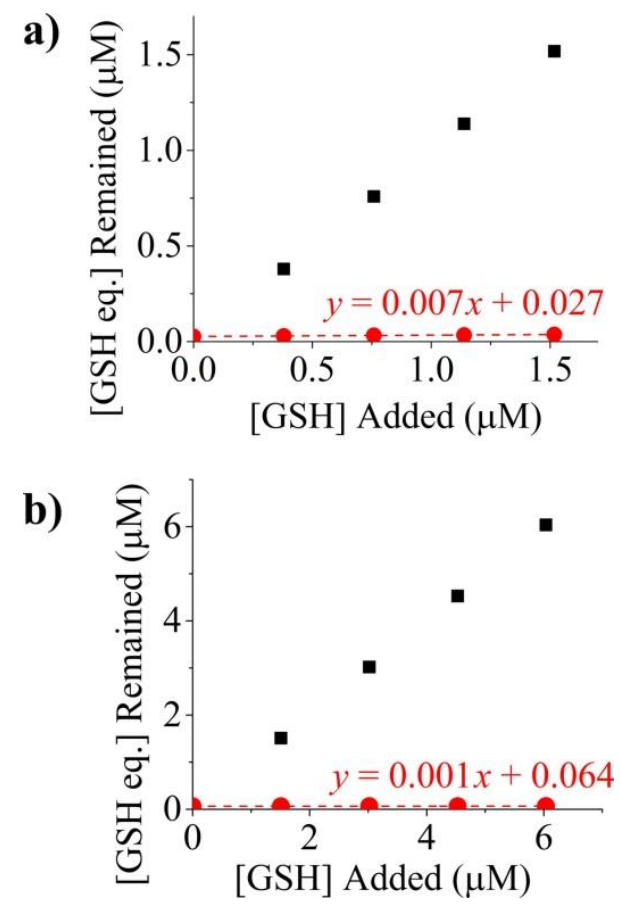

Figure 2: Validation of the GSSG $\left(\mathrm{BQ} / \mathrm{TNB}^{2-}\right)$ assay in a) plasma, and b) saliva. The black squares $(\mathbf{\square})$ and red circles $(\bullet)$ designate the amounts of GSH expected without the masking by BQ/TNB ${ }^{2-}$ and the GSH measured with the GSSG $\left(\mathrm{BQ} / \mathrm{TNB}^{2-}\right)$ assay respectively. The equations displayed in the graphs are those for the lines of best-fit of the GSH concentrations measured with the GSSG (BQ/TNB ${ }^{2-}$ ) assay as a function of GSH added. The slopes of 0.007 and 0.001 give the errors of the measurements of $0.7 \%$ and $0.1 \%$ for plasma and saliva respectively.

The GSSG assay has been further validated to be highly specific to GSH that other thiols in biological samples, cysteine and homocysteine, give rise to an undetectable change in the slope of $\mathrm{A}_{412}$ against time $\left({ }^{d \mathrm{~A}_{412} / d t}\right)$; results not shown. The total glutathione assay has been validated for its specificity and selectivity towards glutathione in our previous paper. ${ }^{26}$

For practical applications, the recommended protocol of the $G S S G\left(B Q / T N B^{2-}\right)$ assay for plasma and saliva samples is summarized in Scheme 1. The protocol describes the reagents employed, the assay for the masking of GSH as well as the assay for enzymatic measurement of glutathione. 
Scheme 1: Protocol of $G S S G\left(B Q / T N B^{2-}\right)$ assay for plasma and saliva samples describing the concentrations of the reagents recommended and the procedures for the masking of GSH as well as the practice of the enzymatic measurement

\section{Reagents}

\begin{tabular}{|c|c|c|c|}
\hline KPE & $\begin{array}{l}0.327 \mathrm{~g} \text { EDTA } \\
100 \mathrm{~mL} \text { PBS pH } 7.5\end{array}$ & BQ & $\begin{array}{l}1.0 \mathrm{mg} \mathrm{BQ} \\
5.0 \mathrm{~mL} \mathrm{KPE}\end{array}$ \\
\hline NADPH & $\begin{array}{l}2.0 \mathrm{mg} \mathrm{NADPH} \\
3.0 \mathrm{~mL} \mathrm{KPE}\end{array}$ & GR & $\begin{array}{l}40 \mu \mathrm{L} \mathrm{GR} \\
3.0 \mathrm{~mL} \mathrm{KPE}\end{array}$ \\
\hline DTNB & $\begin{array}{l}2.0 \mathrm{mg} \text { DTNB } \\
3.0 \mathrm{~mL} \mathrm{KPE}\end{array}$ & & \\
\hline [NB & $\begin{array}{l}1 \mathrm{mg} \mathrm{NaBH}_{4} \\
300 \mu \mathrm{L} \mathrm{DTNB}^{2}\end{array}$ & $\begin{array}{l}\text { wait u } \\
\text { disapp } \\
300 \mu \mathrm{l}\end{array}$ & $\begin{array}{l}\text { Intil bubbles } \\
\text { pear } \\
\text { L KPE }\end{array}$ \\
\hline
\end{tabular}

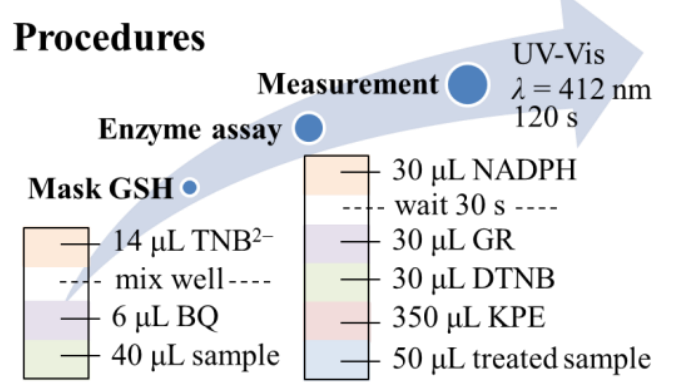

\section{Recovery of glutathione added to human plasma and saliva samples}

The amounts of GSH and GSSG were measured in vitro. The concentrations of both species, however, have been reported to change with storage time. ${ }^{6,39}$ Plasma GSH, in particular, has been reported to decay at a very fast rate at room temperature with a half-life of ca. 20 min. ${ }^{6,39}$ The fast changes in GSH and GSSG concentrations in plasma potentially result in serious in-vitro artefacts. For saliva, on the other hand, there is no literature report on the stability of GSH and GSSG at room temperature. Although there are data showing that salivary GSH is stable for up to 30 days at $-20{ }^{\circ} \mathrm{C} .{ }^{23}$ Therefore, in order to study the stability of glutathione in saliva samples at room temperature as well as to investigate the effect of the medium (plasma vs. saliva) on the rates of changes in GSH and GSSG concentrations, recovery studies are performed next. The recovery study further highlights the key advantage of the short experimental time of the developed GSSG $\left(\mathrm{BQ} / \mathrm{TNB}^{2-}\right)$ assay. In contrast to the other existing enzymatic techniques for the measurement of GSH and GSSG, the newly 
developed $\mathrm{BQ} / \mathrm{TNB}^{2-}$ assay can be completed in $2 \mathrm{~min}$ and hence are suitable for uses in short timescale study.

In this study, plasma and saliva samples were spiked with the solutions of GSH to yield the initial GSH concentrations (at $t=0$ ) of $15 \mu \mathrm{M}$ and $100 \mu \mathrm{M}$ in the spiked samples. The samples were then incubated at $25{ }^{\circ} \mathrm{C}$ and the concentrations of total glutathione, GSH and GSSG were determined every $10 \mathrm{~min}$ for the total time of $90 \mathrm{~min}$. Note that the concentrations presented here are corrected for the concentrations of GSH and GSSG initially present in the sample, i.e. those initially present in the samples are treated as background concentrations and have been set to zero.

In the following discussions, we give values of the concentrations of total glutathione, GSH and GSSG after incubation at $25^{\circ} \mathrm{C}$ for $20 \mathrm{~min}$. The full set of concentrations measured every $10 \mathrm{~min}$ for the total of $90 \mathrm{~min}$ are presented as 'normalized' concentrations in the scattered plots in Figure 3 for a clear comparison between the plasma and saliva (raw data in SI), and between the different concentrations studied. The normalized concentration ( $c$ normalized $)$ is the concentration measured at time $t\left(c_{(t)}\right)$ divided by the initial concentration $\left(\mathrm{c}_{(t=0)}\right)$; $c_{\text {normalized }}=c_{(t)} / \mathrm{c}_{(t=0)}$. All concentrations are given as GSH equivalents which are equal to $[\mathrm{GSH}]+2[\mathrm{GSSG}]$ and $2[\mathrm{GSSG}]$ for total glutathione and oxidized glutathione respectively.

Plasma. The concentrations of plasma glutathione change rapidly at both initial GSH concentrations studied $(15 \mu \mathrm{M}$ and $100 \mu \mathrm{M})$. At $15 \mu \mathrm{M}$ initial GSH concentration after incubation at $25{ }^{\circ} \mathrm{C}$ for $20 \mathrm{~min}$, the concentrations of total glutathione and GSH drop to $41 \%$ and $36 \%$, while GSSG concentration rises to $5 \%$. For the initial GSH of $100 \mu \mathrm{M}$, the concentrations of total glutathione and GSH are $73 \%$ and $60 \%$ after 20 min incubation respectively, while GSSG increases to $13 \%$. 
Saliva. The levels of salivary total glutathione, GSH and GSSG change very slowly with time. The total glutathione and GSH levels are stable within the errors of $\pm 6 \%$ and $\pm 3 \%$ for the initial GSH concentrations of $15 \mu \mathrm{M}$ and $100 \mu \mathrm{M}$ in the first 20 min of incubation. After 20 min, the changes in concentrations of total glutathione and GSH become more significant, reaching up to $20 \%$ after incubation for $90 \mathrm{~min}$, the change of which is nonetheless much smaller than in plasma.

The significantly greater stability of saliva at room temperature $\left(25^{\circ} \mathrm{C}\right)$ offers the advantage of more reliable clinical information regarding antioxidant and the oxidative status of the cells compared to the information obtained from plasma currently used for the diagnosis.

\section{Plasma}

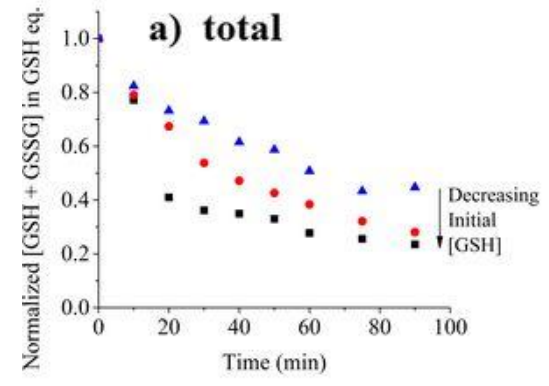

d) total

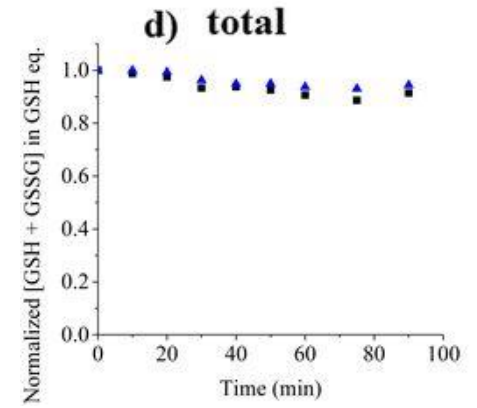

b) reduced

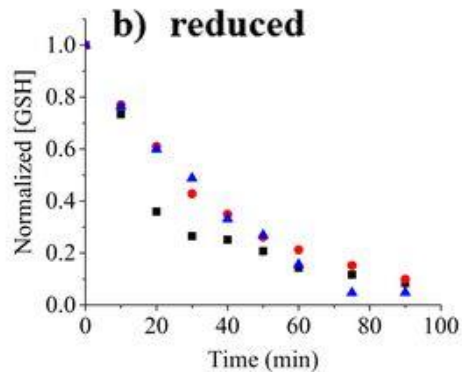

Saliva

e) reduced

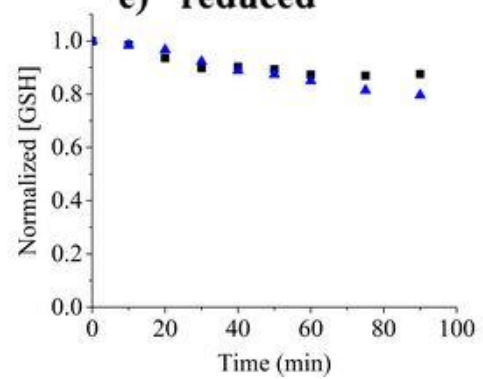

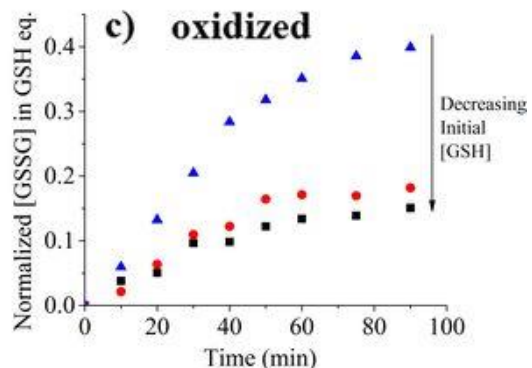

f) oxidized

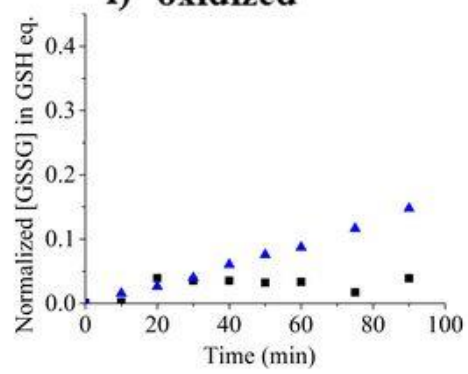

Figure 3: Plasma: changes in normalized concentrations $\left(c_{\text {normalized }}=c_{(t)} / c_{(t=0)}\right)$ of a) total glutathione $(\mathrm{GSH}+$ GSSG), b) reduced glutathione (GSH) and c) oxidized glutathione (GSSG) as a function of time at different initial GSH concentrations spiked in plasma; blue: $100 \mu \mathrm{M}$, red: $50 \mu \mathrm{M}$, black: $15 \mu \mathrm{M}$. Saliva: changes in normalized concentrations of $\mathbf{d}$ ) total glutathione (GSH + GSSG), e) reduced glutathione (GSH) and f) oxidized glutathione (GSSG) as a function of time at different initial GSH concentrations spiked in saliva; blue: $100 \mu \mathrm{M}$, black: $15 \mu \mathrm{M}$. 
The results of the stability studies presented in Figure 3 further show that the concentration of GSSG increases as a function of time, while the concentration of GSH decreases, suggesting there is a conversion of GSH to GSSG. However, the concentration of total glutathione also decreases with time. This implies that not all the consumption of GSH is due to the formation of GSSG. Consequently, to further explore the stability of GSH and GSSG in the samples, we additionally observed the speciation of the products at different initial GSH concentrations studied. In saliva, the speciation of the products after incubation at $25{ }^{\circ} \mathrm{C}$ for $90 \mathrm{~min}$, described in terms of the ratio of GSSG to other products, changes from 0.4 (4\%: $9 \%$ ) to 2.5 (15\%:6\%) for the initial concentration changes from $15 \mu \mathrm{M}$ to $100 \mu \mathrm{M}$. On the other hand, the speciation changes of the products in plasma are $0.2(15 \%: 77 \%)$ and $0.7(40 \%: 55 \%)$ at the initial GSH concentrations of $15 \mu \mathrm{M}$ and $100 \mu \mathrm{M}$ respectively.

This result may be explained by the two possible routes of the loss of GSH from the system. First is the oxidation of GSH to GSSG catalyzed by metal catalysts such as copper(II) and iron(III). ${ }^{30}$ Second is the degradation of GSH into its amino acid constituents (glutamic acid, cysteine and glycine) catalyzed by the enzyme $\gamma$-glutamyltransferase (GGT). ${ }^{40}$ The concentrations of copper in plasma and saliva have been reported to be ca. $20 \mu \mathrm{M}^{41-43}$ and $1 \mu \mathrm{M}^{44}$ respectively, although there are papers reporting a wide range of copper contents in saliva from $0.8 \mu \mathrm{M}$ to $12.0 \mu \mathrm{M} .^{45,46}$ Salivary and serum GGT activities have been reported to be $2.9 \pm 1.0$ units/L and $14.6 \pm 5.8$ units/L respectively. ${ }^{47}$ The levels of copper as well as the activities of GGT in the two media are consistent with the results that salivary glutathione is, on the basis of these results, more stable than plasma. Consequently, this information together with the variable speciation of products observed support the hypotheses that both metal and biological catalysts play important roles in the loss of GSH as well as total glutathione from biological samples. 
In addition to copper and GGT, the formation of mixed protein disulfides may also contribute to the loss of glutathione from biological samples. The total protein content in plasma $(68.5$ $\pm 1.0 \mathrm{mg} / \mathrm{mL})^{48}$ is two orders of magnitude higher than in saliva $(0.68 \pm 0.07 \mathrm{mg} / \mathrm{mL}),{ }^{49}$ consistent with the fact that the loss of glutathione in plasma happens much more rapidly than in saliva. However, note that the concentration of glutathione decreases rapidly in plasma even when the samples are deproteinized, suggesting the significant roles of copper and GGT. $^{6}$

After all, the results presented herein display potential problems in accurately determining the levels of glutathione in biological samples due to the in-vitro artefacts. At the same time, the results show that the problem is minimized in saliva, and hence emphasize that saliva offers greater advantage than plasma in terms of the stability of GSH and GSSG in addition to its non-invasive nature.

\section{Conclusions}

Benzoquinone (BQ) has been introduced as a new, rapid thiol-masking reagent for uses in the quantification of GSH and GSSG via the enzymatic recycling assay. Excess BQ is removed by the rapid reaction with $\mathrm{TNB}^{2-}$ (made from the reduction of DTNB by $\mathrm{NaBH}_{4}$ ), exploiting the selectivity of glutathione reductase. This developed assay can be completed within 2 min, in contrast to other techniques which require at least $30 \mathrm{~min}$ for reaction to go to completion (e.g. 2VP), or for the efforts required to remove excess chemical (e.g. NEM). The assay can be directly applied to biological samples such as plasma and saliva. The $\mathrm{BQ} / \mathrm{TNB}^{2-}$ assay is thus a significant improvement to the enzymatic cycling assay, which is the most widely-used technique for determination of glutathione. The new assay has been demonstrated to be particularly useful in situations where limited time is available for accurate analysis such as the diagnosis of plasma glutathione, the level of which decreases significantly with the half- 
life of ca. 5-20 min (half-life decreases with initial concentration), or the kinetic/stability study of glutathione levels in biological samples, such as the work done in this paper. From this demonstration of application of the developed assay, we have further shown that the use of saliva is preferential for the determination of GSH, GSSG and the oxidative status in terms of the stability and the accurate representation of the 'true' values of these parameters in the body (i.e. minimized in-vitro effect in saliva than plasma) in addition to the non-invasive nature of its sampling.

\section{Acknowledgements}

KN thanks the Royal Thai government (DPST scholarship) for funding. KN, CBM and RGC are supported by funding from the European Research Council under the European Union's Seventh Framework Programme (FP/2007-2013)/ERC Grant Agreement no. [320403].

\section{References}

(1) Sies, H. Oxidative Stress; Elsevier Science, 2013.

(2) Pastore, A.; Federici, G.; Bertini, E.; Piemonte, F. Clin. Chim. Acta 2003, 333, 19-39.

(3) Schulz, J. B.; Lindenau, J.; Seyfried, J.; Dichgans, J. Eur. J. Biochem. 2000, 267, 4904-4911.

(4) Mills, B. J.; Weiss, M. M.; Lang, C. A.; Liu, M. C.; Ziegler, C. J. Lab. Clin. Med. 2000, 135, 396-401.

(5) Throughout this paper, the glutathione enzymatic recycling assay developed by Tietze is referred to as the total glutathione assay. Meanwhile, the modified assay utilizing the thiolmasking reagent developed in this work for the detection of oxidized glutathione is referred to as the $G S S G\left(B Q / T N B^{2-}\right)$ assay.

Abbreviations: GR, glutathione reductase; NADPH, nicotinamide adenine dinucleotide phosphate; GSH, reduced glutathione; GSSG, oxidized glutathione; DTNB, 5,5'dithiobis(2-nitrobenzoic acid); $\mathrm{TNB}^{2-}$, 5-thio-2-nitrobenzoate; $\mathrm{BQ}$, benzoquinone; 2VP, 2vinylpyridine; NEM, $N$-ethylmaleimide; GGT, gamma-glutamyl transferase.

(6) Beutler, E.; Duron, O.; Kelly, B. M. J. Lab. Clin. Med. 1963, 61, 882-888.

(7) Reed, D. J.; Babson, J. R.; Beatty, P. W.; Brodie, A. E.; Ellis, W. W.; Potter, D. W. Anal. Biochem. 1980, 106, 55-62.

(8) Neuschwander-Tetri, B. A.; Roll, F. J. Anal. Biochem. 1989, 179, 236-241. 
(9) Serru, V.; Baudin, B.; Ziegler, F.; David, J. P.; Cals, M. J.; Vaubourdolle, M.; Mario, N. Clin. Chem. 2001, 47, 1321-1324.

(10) Carru, C.; Zinellu, A.; Mario Pes, G.; Marongiu, G.; Tadolini, B.; Deiana, L. Electrophoresis 2002, 23, 1716.

(11) Harfield, J. C.; Batchelor-McAuley, C.; Compton, R. G. The Analyst 2012, 137, 2285.

(12) Lee, P. T.; Goncalves, L. M.; Compton, R. G. Sens. Actuators, B 2015, 221, 962-968.

(13) Zaidi, S. A.; Shin, J. H. Anal. Methods 2016, 8, 1745-1754.

(14) Chen, Z.; Wang, Z.; Chen, J.; Wang, S.; Huang, X. The Analyst 2012, 137, 31323137 .

(15) Han, H.-Y.; He, Z.-K.; Zeng, Y.-E. Microchim. Acta 2006, 155, 431-434.

(16) Monostori, P.; Wittmann, G.; Karg, E.; Turi, S. J. Chromatogr. B Analyt. Technol. Biomed. Life Sci. 2009, 877, 3331-3346.

(17) Camera, E.; Picardo, M. J. Chromatogr. B 2002, 781, 181-206.

(18) Tietze, F. Anal. Biochem. 1969, 27, 502-522.

(19) Griffith, O. W. Anal. Biochem. 1980, 106, 207-212.

(20) Güntherberg, H.; Rost, J. Anal. Biochem. 1966, 15, 205-210.

(21) Giustarini, D.; Dalle-Donne, I.; Milzani, A.; Fanti, P.; Rossi, R. Nat. Protoc. 2013, 8, 1660-1669.

(22) Richie, J. P., Jr.; Skowronski, L.; Abraham, P.; Leutzinger, Y. Clin. Chem. 1996, 42, 64-70.

(23) Emekli-Alturfan, E.; Yarat, A.; Caliskan-Ak, E.; Pisiriciler, R.; Kuru, B.; Noyan, U. J. Clin. Lab. Anal. 2013, 27, 261-266.

(24) Riddles, P. W.; Blakeley, R. L.; Zerner, B. Methods Enzymol. 1983, 91, 49-60.

(25) Henderson, G. B.; Murgolo, N. J.; Kuriyan, J.; Osapay, K.; Kominos, D.; Berry, A.; Scrutton, N. S.; Hinchliffe, N. W.; Perham, R. N.; Cerami, A. Proc. Natl. Acad. Sci. U. S. A. 1991, 88, 8769-8773.

(26) Ngamchuea, K.; Batchelor-McAuley, C.; Cowen, P. J.; Williams, C.; Goncalves, L. M.; Compton, R. G. The Analyst 2016, 141, 4707-4712.

(27) Eckert, K. G.; Eyer, P.; Sonnenbichler, J.; Zetl, I. Xenobiotica 2008, 20, 351-361.

(28) Lin, Q.; Li, Q.; Batchelor-McAuley, C.; Compton, R. G. J. Phys. Chem. C 2015, 119, 1489-1495.

(29) Long, G. L.; Winefordner, J. D. Anal. Chem. 1983, 55, 712A-724A.

(30) Ngamchuea, K.; Batchelor-McAuley, C.; Compton, R. G. Chem. - Eur. J. 2016, 22, 15937-15944.

(31) O'Brien, P. J. Chem. Biol. Interact. 1991, 80, 1-41.

(32) Brunmark, A.; Cadenas, E. Chem. Biol. Interact. 1988, 68, 273-298.

(33) Stewart, A. J.; Blindauer, C. A.; Berezenko, S.; Sleep, D.; Tooth, D.; Sadler, P. J. FEBS J. 2005, 272, 353-362.

(34) Kanchuger, M. S.; Byers, L. D. J. Am. Chem. Soc. 1979, 101, 3005-3010.

(35) Rahman, I.; Kode, A.; Biswas, S. K. Nat. Protoc. 2006, 1, 3159-3165.

(36) Michelet, F.; Gueguen, R.; Leroy, P.; Wellman, M.; Nicolas, A.; Siest, G. Clin. Chem. 1995, 41, 1509-1517.

(37) Zappacosta, B.; Manni, A.; Persichilli, S.; Boari, A.; Scribano, D.; Minucci, A.; Raffaelli, L.; Giardina, B.; De Sole, P. Clin. Biochem. 2007, 40, 661-665. 
(38) Iwasaki, Y.; Hoshi, M.; Ito, R.; Saito, K.; Nakazawa, H. J. Chromatogr. B Analyt. Technol. Biomed. Life Sci. 2006, 839, 74-79.

(39) Jones, D. P.; Carlson, J. L.; Samiec, P. S.; Sternberg, P., Jr.; Mody, V. C., Jr.; Reed, R. L.; Brown, L. A. Clin. Chim. Acta 1998, 275, 175-184.

(40) Whitfield, J. B. Crit. Rev. Clin. Lab. Sci. 2001, 38, 263-355.

(41) Ward, A. F.; Mitchell, D. G.; Kahl, M.; Aldous, K. M. Clin. Chem. 1974, 20, 11991203.

(42) Aldor, Y.; Walach, N.; Modai, D.; Horn, Y. Klin. Wochenschr. 1982, 60, 375-377.

(43) Kurekci, A. E.; Alpay, F.; Tanindi, S.; Gokcay, E.; Ozcan, O.; Akin, R.; Isimer, A.; Sayal, A. Epilepsia 1995, 36, 600-604.

(44) Ayinampudi, B. K.; Narsimhan, M. J. Oral Maxillofac. Pathol. 2012, 16, 178-182.

(45) Dreizen, S.; Spies, H. A., Jr.; Spies, T. D. J. Dent. Res. 1952, 31, 137-142.

(46) Dejorge, F. B.; Canelas, H. M.; Dias, J. C.; Cury, L. Clin. Chim. Acta 1964, 9, 148150 .

(47) Jiménez-Alonso, J.; Sabio, J. M.; Rivera-Cívico, F.; Martín-Armada, M. a.; Rodríguez, M. Á.; Jáimez, L.; Castillo, M. a. J.; Sánchez-Román, J.; Castro, J. Clin. Chim. Acta 2003, 334, 225-231.

(48) Morris, B.; Courtice, F. C. Q. J. Exp. Physiol. Cogn. Med. Sci. 1955, 40, 127-137.

(49) Walsh, N. P.; Blannin, A. K.; Clark, A. M.; Cook, L.; Robson, P. J.; Gleeson, M. J. Sports Sci. 1999, 17, 129-134. 


\section{For TOC Only}

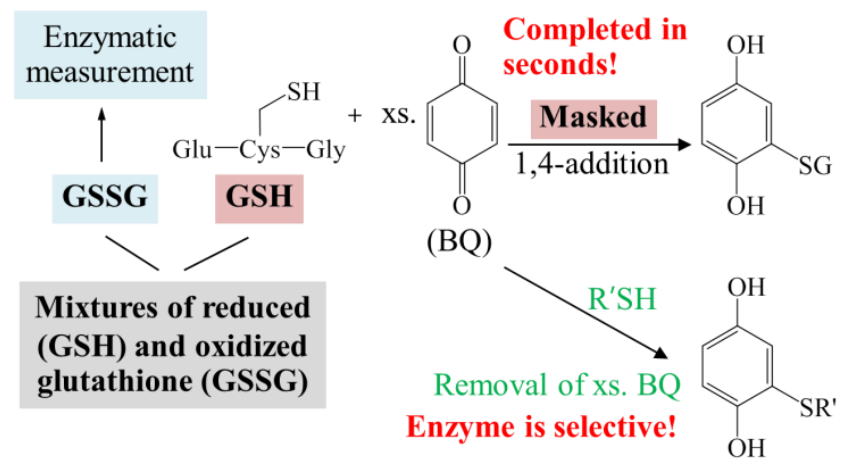

